\title{
ATRX and the replication of structured DNA
}

David Clynes, Richard J. Gibbons

MRC Molecular Haematology Unit, Weatherall Institute of Molecular Medicine, University of Oxford, John Radcliffe Hospital, Oxford OX3 9DS, UK 


\section{Summary}

Understanding the underlying molecular basis for disease can often be a prolonged and tortuous process with many false leads and blind alleys. Relating the cause of ATR-X syndrome to the function of the protein ATRX is a case in point. In this review we attempt to bring together the diverse biological phenomena associated with ATRX dysfunction with what has recently been discovered concerning the chromatin remodeling activity of this protein. This potentially casts light on how defective DNA replication/histone replacement can impact on transcription, telomere maintenance and also possibly chromosome segregation.

\section{Introduction}

Germ line mutations in ATRX give rise to a complex human genetic disease, $\mathrm{X}$ linked alpha thalassemia mental retardation (ATR-X) syndrome characterized by severe learning difficulties, a characteristic facial appearance, abnormal sexual development and a form of anemia, alpha thalassemia [1]. The latter arises because of reduced expression of alpha globin, a component of adult hemoglobin. Originally this transcriptional defect was a defining feature of the condition and the working hypothesis was of an $\mathrm{X}$ encoded protein facilitating the expression of a wide repertoire of genes. Subsequently, in numerous different animal models, perturbation of ATRX has been associated with a wide range of effects: altered patterns of DNA methylation (at subtelomeres, hetrochromatic repeats and ribosomal DNA) [2], aberrant chromosome congression in mitosis [3] and segregation in meiosis, [4] as well as telomere dysfunction $\left[5^{\star *}\right]$. Is there a central defect which might unify these disparate effects? 
At first glance these effects at least relate to where ATRX localizes in the genome predominantly large tandemly repeated regions such as ribosomal DNA, pericentric heterochromatin [6] and telomeres $\left[5^{* *}, 7^{* *}\right]$. In mouse embryonic stem (ES) cells ATRX is recruited to telomeres during $S$ phase. If ATRX is depleted there is an increase in the presence of $\gamma \mathrm{H} 2 \mathrm{AX}$, a mark of replicative stress, at these telomeric foci $\left[5^{\star *}\right]$. This important observation strongly suggests that ATRX is playing a role in maintaining telomere integrity and given its presence during DNA synthesis it is likely that it facilitates normal telomere replication. This role for ATRX was strengthened by the findings of Huh et al., who showed that in mouse myoblasts ATRX co-localises with late replicating chromatin during $S$ phase and when ATRX is knocked out progression through late $S$ phase is delayed, there is a DNA damage response and telomere fragility is observed [8*].

Mammalian telomeres consist of many kilobases of the tandem repeat (TTAGGG)n. DNA replication is unidirectional commencing in the subtelomeric region and proceeding to the telomere ends [9]. Tandem repeats have the potential to fold into non B-forms of DNA (eg cruciform, triplex and hairpin secondary structures) [10]. Grich repeats can form $\mathrm{G}$ quadruplex $(\mathrm{G} 4)$ structures and there is recent evidence that these structures exist in vivo [11-13] with perhaps the best evidence at telomeres $[9,14]$. Many ATRX targets are predicted to form G4 [15**] and electrophoretic mobility shift assays have demonstrated that recombinant ATRX protein preferentially binds to G4 structured DNA over the unfolded G4 telomere derived sequence $\left[15^{* *}\right]$. G4 (and other secondary structures) are particularly likely to form if 
the DNA is single stranded (ssDNA). At telomeres, the lagging strand during DNA replication and non-template strand during transcription are both G-rich and single stranded and hence offer likely substrates for G4 formation. One compelling hypothesis is that the presence of such DNA secondary structures may perturb DNA replication and that at certain tandem repeats ATRX is required to facilitate their resolution or bypass. How might this relate to the chromatin remodeling activity of ATRX?

\section{Chromatin remodelling activity of ATRX}

We now know that ATRX plays an important role in the incorporation of the histone variant $\mathrm{H} 3.3$ into telomeric, ribosomal and pericentromeric DNA and this role may extend to the smaller interstitial repeats $[7,16]$. ATRX works with its partner protein DAXX, the latter of which directly binds $\mathrm{H} 3.3$ and acts as a histone chaperone, whereas ATRX both directs where this occurs and facilitates the incorporation of H3.3 into chromatin [17]. Unlike the canonical histones, the incorporation of which is closely coupled to the progression of the replication fork, histone $\mathrm{H} 3.3$ is predominantly incorporated into chromatin in a replication-independent manner and appears principally to serve as a replacement variant $[18,19]$.

One might envisage a number of ways by which ATRX helps maintain the integrity of telomere chromatin. Repetitive DNA is predicted to be an inherently unfriendly substrate for nucleosomes leading to rapid turnover [20] and a replication independent mechanism for histone replacement is therefore needed, particularly in post mitotic, terminally differentiated cells. Consistent with this, H3.3 is also 
associated with actively transcribed regions where nucleosome occupancy is also known to be highly dynamic [21]. In mouse ES cells, however, H3.3 is deposited at telomeres by ATRX/DAXX predominantly during $S$ phase, which at first sight appears paradoxical for a histone variant associated with replication independence $\left[5^{\star *}\right]$. Is H3.3 acting as a replacement histone variant during S phase?

In human fibroblasts there is evidence for frequent stalling of replication forks, detectable by RPA34 accumulation at long tracks of ssDNA formed as a result of uncoupling of the replicative helicase and DNA polymerization [22]. The telomere specific recruitment of the low fidelity enzyme DNA polymerase $\beta$ at the same time suggests that repair is associated with DNA lesion bypass. Since this repair is dissociated from the replication fork it will also be uncoupled from replicationdependent histone deposition. In order to preserve the chromatin integrity a replication independent mechanism for histone deposition is required [23]. Wong et al. $\left[5^{* *}\right]$ showed that in mouse ES cells ATRX and H3.3 localised to telomeres at a comparable stage of $S$ phase so it is tempting to think that ATRX/DAXX is depositing H3.3 during post-replicative repair rather than at the replication fork. This is consistent with the observations of Ray-Gallet et al. [19] who showed that although H3.3 is incorporated into chromatin during S phase the sites do not overlap with replication sites. In the absence of ATRX one might envisage that the replication stress observed could be related either to the failure to chromatinise the repaired DNA or the failure to restart a stalled fork.

\section{Abnormal gene expression in ATR-X syndrome}


How might such a problem lead to the perturbation in gene expression seen in ATR$\mathrm{X}$ syndrome? Although the localization of ATRX to large structural repeats can be seen by fluorescent microscopy, chromatin immunoprecipitation of ATRX reveals the finer detail showing that ATRX also localises to GC rich sequences including CpG islands and GC-rich interstitial repeats [15**]. Looking at the well characterised end of chromosome 16 (16p13.3), wherein lies the cluster of alpha-like globin genes, it was noted that the peak of binding was at a variable number tandem repeat (VNTR), $\psi \zeta$, within the cluster (Figure 1a). In patients with ATR-X syndrome, it was observed that the globin genes closest to the repeat were the most down regulated, and those furthest away ( 10kb) least affected (Figure 1b). Furthermore, the larger the VNTR the more severe was the degree of down-regulation, even in individuals with the same ATRX mutation (Figure 1c). The same pattern was noted at another VNTR about $300 \mathrm{~kb}$ away from the alpha cluster. The associated gene, NME4 and its near neighbor DECR2 were both affected, but NME4, which contains the VNTR, was more severely affected. Again, the degree of down-regulation was related to the size of the VNTR that lies in cis. This observation helps explain the characteristics of the genes affected in ATR-X syndrome - they happened to be located near an ATRXbinding G-rich repeat. It also explains the variable phenotypes, or at least the severity of the alpha thalassemia seen in ATR-X patients. Like the telomeres, the sequences of these repeats are able to form G4 in vitro so it is tempting to think that ATRX is acting to relieve the effects of G4 at these loci. Might the underlying problem again be with DNA replication, with the effect on local gene an associated epiphenomenon? Evidence for a link between a defect in replicating structured DNA and the perturbation of local gene expression has been demonstrated by Sarkies et 
al. [23, 24*]. Using avian DT40 cells they showed that REV1, a low fidelity polymerase involved in translesional DNA synthesis, is important in replicating G4 DNA. In its absence there is an uncoupling of replication dependent histone deposition and DNA synthesis at the replication fork. As a consequence of this the pre-existing histones with their epigenetic marks were no longer efficiently recycled to the daughter strands and this leads to the loss of epigenetic memory in the region and consequently a change in local gene expression. Where previously gene expression was repressed, the genes were activated and conversely where genes were actively expressed they became silenced. Given that stalled replication forks have the propensity to collapse and generate double strand breaks, a compelling alternative explanation is that the associated silencing effects arise from epigenetic modifcations originating from the site of a break. Indeed, work by Shanbhag et al., demonstrate that the induction of a double strand break can lead to the silencing of a reporter gene up to $4 \mathrm{~kb}$ away from the break site through $\mathrm{H} 2 \mathrm{~A}$ ubiquitylation dependent pausing of RNAPII [25]. We propose that in the absence of ATRX there is a defect replicating the structured DNA associated with these G-rich tandem repeats and this may trigger fork collapse and break associated epigenetic silencing. Alternatively this may lead to the uncoupling of replication dependent histone deposition and the subsequent loss of activating histone marks at the alpha globin loci and reduced alpha globin expression causing alpha thalassaemia.

\section{ATRX and the Alternative Lengthening of Telomere (ALT) pathway}


Of considerable recent interest is the finding that ATRX mutations are often associated with a growing group of cancer types. In order to divide indefinitely tumour cells must have a mechanism to maintain the length of their telomeres, without which they will shorten progressively with every round of DNA replication eventually leading to senescence or apoptosis [26]. In the majority of cancers, this shortening is counteracted by the expression of telomerase, a specialized reverse transcriptase which adds TTAGGG repeats; normally telomerase is only expressed in stem cells and germ cells. In $10-15 \%$ of cancers the telomeres are maintained by a telomerase independent mechanism known as the alternative lengthening of telomeres pathway (ALT) [27]. This pathway depends on homologous recombination involving either other telomeres or the extra chromosomal telomeric repeats which are a feature of ALT tumours [28]. Inactivating mutations in ATRX or DAXX have been identified in many ALT cancers and in a comprehensive study the loss of ATRX was observed in $90 \%$ of ALT cell lines [29-31*]. The recent identification of histone H3.3 mutations in addition to ATRX/DAXX mutations in paediatric gliablastomas and their correlation with ALT characteristics has reinforced this association [32] and strongly suggest that ATRX/DAXX/H3.3 normally suppresses this pathway. Depletion of ATRX appears insufficient to induce ALT suggesting that the effect is context specific $\left[31^{*}\right]$. A study in pancreatic neuroendocrine tumours arising in individuals with multiple endocrine neoplasia-1 (MEN-1) syndrome showed that the loss of ATRX and/or DAXX was not apparent until the tumour size was $>3 \mathrm{~cm}$, suggesting these are late events in tumour development [33]. It is possible that mutations in ATRX/DAXX are permissive to the continued growth of these tumours through the triggering of the ALT pathway. It is interesting to note that in one 
paediatric glioblastoma three ATRX mutations were identified presumably in independent clones within the tumour [32]. How might the absence of ATRX/DAXX trigger this pathway? One possible model is that in the absence of ATRX, forks stalled by structural DNA are not efficiently processed. The unidirectional nature of replication at telomeres [9] means that stalled forks cannot be salvaged by a converging replication fork. In these circumstances fork restart is therefore solely dependent on $\mathrm{HR}$, and this triggering of $\mathrm{HR}$ now provides a pathway for telomere maintenance and permits further tumour growth (Figure 2).

\section{Concluding remarks}

Defining the chromatin remodeling activity of ATRX has been a great step forward in understanding the function of this protein. The next challenge which has been outlined in this review is to explain the diverse phenomena associated with germline and acquired mutations in ATRX. Its role in the replication of telomeres has stimulated a surge of interest in its biology and it is to be expected that new and exciting leads to determining ATRX function will come thick and fast.

\section{Box 1. ATRX in Meiosis and mitosis}

It is tempting to speculate that, in addition to triggering replication and transcriptional silencing defects, a failure to properly chromatinise heterochromatic regions through the deposition of $\mathrm{H} 3.3$ could also be accountable for the chromosomal instability defects observed upon loss of ATRX function. RNAi induced knockdown of ATRX in mouse oocytes generates a range of chromosome segregation defects during the metaphase II stage of meiosis, including impaired chromosome alignment and loss 
of sister chromatid cohesion [4] with similar abnormalities observed in mouse myoblasts in which ATRX is knocked out [8*]. Consistent with this, faithful mitotic chromosome cohesion and congression is also dependent on ATRX function [3]. Of note studies in drosophila have shown that $\mathrm{H} 3.3$ is also required for faithful meiotic chromosome segregation in male germ cells [34], lending weight to the idea that a loss of H3.3 deposition could at least partially account for the ATRX associated phenotypes. Indeed, ATRX has been shown to be required for the recruitment of the H3.3 chaperone DAXX to pericentromeric heterochromatin domains in the female germline [35], lending credence to such a model.

\section{References and recommended reading}

Papers of particular interest, published within the period of review, have been highlighted as:

- of special interest

* of outstanding interest

\section{Acknowledgements}

The authors would like to thank Doug Higgs for his continued support and encouragement. This work was supported by the Medical Research Council and the University of Oxford.

\section{References}


1. Gibbons RJ, Picketts DJ, Higgs DR: Syndromal mental retardation due to mutations in a regulator of gene expression. Human Molecular Genetics 1995, 4:1705-1709.

2. Gibbons RJ, McDowell TL, Raman S, O'Rourke DM, Garrick D, Ayyub H, Higgs DR: Mutations in ATRX, encoding a SWI/SNF-like protein, cause diverse changes in the pattern of DNA methylation. Nat Genet 2000, 24:368-371.

3. Ritchie K, Seah C, Moulin J, Isaac C, Dick F, Berube NG: Loss of ATRX leads to chromosome cohesion and congression defects. J Cell Biol 2008, 180:315-324.

4. De La Fuente R, Viveiros MM, Wigglesworth K, Eppig JJ: ATRX, a member of the SNF2 family of helicase/ATPases, is required for chromosome alignment and meiotic spindle organization in metaphase II stage mouse oocytes. Dev Biol 2004, 272:1-14.

**5. Wong LH, McGhie JD, Sim M, Anderson MA, Ahn S, Hannan RD, George AJ, Morgan KA, Mann JR, Choo KH: ATRX interacts with H3.3 in maintaining telomere structural integrity in pluripotent embryonic stem cells. Genome Res 2010, 20:351-360.

First study to demonstrate the recruitment of ATRX to telomeres during S-phase in mouse embryonic stem (ES) cells and that RNAi mediated knockdown of ATRX induces a telomere dysfunction.

6. McDowell TL, Gibbons RJ, Sutherland H, O'Rourke DM, Bickmore WA, Pombo A, Turley H, Gatter K, Picketts DJ, Buckle VJ, et al.: Localization of a putative transcriptional regulator (ATRX) at pericentromeric heterochromatin and 
the short arms of acrocentric chromosomes. Proc Natl Acad Sci U S A 1999, 96:13983-13988.

**7. Goldberg AD, Banaszynski LA, Noh KM, Lewis PW, Elsaesser SJ, Stadler S, Dewell S, Law M, Guo X, Li X, et al.: Distinct factors control histone variant H3.3 localization at specific genomic regions. Cell 2010, 140:678691.

Study characterising the genome wide localisation of the histone variant $\mathrm{H} 3.3$ in mouse ES and neuronal precursor cells. The authors demonstrate that ATRX is required for the deposition of $\mathrm{H} 3.3$ at telomeres.

*8. Huh MS, Price O'Dea T, Ouazia D, McKay BC, Parise G, Parks RJ, Rudnicki MA, Picketts DJ: Compromised genomic integrity impedes muscle growth after Atrx inactivation. J Clin Invest 2012.

Study characterising the role of ATRX in myoblast cells showing that ATRX colocalises to late replicating heterochromatin in S phase, with its knockout leading to an elongation in S-phase, accumulation of p53, genomic instability and telomere dysfunction.

9. Sfeir A, Kosiyatrakul ST, Hockemeyer D, MacRae SL, Karlseder J, Schildkraut CL, de Lange T: Mammalian telomeres resemble fragile sites and require TRF1 for efficient replication. Cell 2009, 138:90-103.

10. Bacolla A, Wells RD: Non-B DNA conformations as determinants of mutagenesis and human disease. Mol Carcinog 2009, 48:273-285.

11. Lipps HJ, Rhodes D: G-quadruplex structures: in vivo evidence and function. Trends Cell Biol 2009, 19:414-422. 
12. Rodriguez R, Miller KM, Forment JV, Bradshaw CR, Nikan M, Britton S, Oelschlaegel T, Xhemalce B, Balasubramanian S, Jackson SP: Smallmolecule-induced DNA damage identifies alternative DNA structures in human genes. Nat Chem Biol 2012, 8:301-310.

13. Paeschke K, Capra JA, Zakian VA: DNA replication through G-quadruplex motifs is promoted by the Saccharomyces cerevisiae Pif1 DNA helicase. Cell 2011, 145:678-691.

14. Schaffitzel C, Berger I, Postberg J, Hanes J, Lipps HJ, Pluckthun A: In vitro generated antibodies specific for telomeric guanine-quadruplex DNA react with Stylonychia lemnae macronuclei. Proc Natl Acad Sci U S A 2001, 98:8572-8577.

**15. Law MJ, Lower KM, Voon HP, Hughes JR, Garrick D, Viprakasit V, Mitson M, De Gobbi M, Marra M, Morris A, et al.: ATR-X Syndrome Protein Targets Tandem Repeats and Influences Allele-Specific Expression in a SizeDependent Manner. Cell 2010, 143:367-378.

Reports the genome wide distribution of ATRX at tandem repeat sequences (TRs) at both telomeres and euchromatin. Showed tha genes associated with these TRs are often deregulated when ATRX is mutated and the change in expression is associated by the size and proximity of the associated VNTR.

16. Drane P, Ouararhni K, Depaux A, Shuaib M, Hamiche A: The death-associated protein DAXX is a novel histone chaperone involved in the replicationindependent deposition of H3.3. Genes Dev 2010, 24:1253-1265.

17. Lewis PW, Elsaesser SJ, Noh KM, Stadler SC, Allis CD: Daxx is an H3.3specific histone chaperone and cooperates with ATRX in replication- 
independent chromatin assembly at telomeres. Proc Natl Acad Sci U S A 2010, 107:14075-14080.

18. Tagami H, Ray-Gallet D, Almouzni G, Nakatani Y: Histone H3.1 and H3.3 complexes mediate nucleosome assembly pathways dependent or independent of DNA synthesis. Cell 2004, 116:51-61.

19. Ray-Gallet D, Woolfe A, Vassias I, Pellentz C, Lacoste N, Puri A, Schultz DC, Pchelintsev NA, Adams PD, Jansen LE, et al.: Dynamics of histone H3 deposition in vivo reveal a nucleosome gap-filling mechanism for $\mathrm{H} 3.3$ to maintain chromatin integrity. Mol Cell $2011,44: 928-941$.

20. Schneiderman JI, Sakai A, Goldstein S, Ahmad K: The XNP remodeler targets dynamic chromatin in Drosophila. Proc Natl Acad Sci U S A 2009, 106:14472-14477.

21. Ahmad K, Henikoff S: Histone H3 variants specify modes of chromatin assembly. Proc Natl Acad Sci U S A 2002, 99 Suppl 4:16477-16484.

22. Verdun RE, Karlseder J: The DNA damage machinery and homologous recombination pathway act consecutively to protect human telomeres. Cell 2006, 127:709-720.

23. Sarkies P, Reams C, Simpson LJ, Sale JE: Epigenetic instability due to defective replication of structured DNA. Mol Cell 2010, 40:703-713.

*24. Sarkies P, Murat P, Phillips LG, Patel KJ, Balasubramanian S, Sale JE: FANCJ coordinates two pathways that maintain epigenetic stability at Gquadruplex DNA. Nucleic Acids Res 2012, 40:1485-1498.

Showed that interruption of DNA replication at G4 sequences results in loss of the tight coupling between replication and the recycling of parental histones. This leads 
to the biased incorporation of newly synthesized histones and loss of epigenetic memory.

25. Shanbhag NM, Rafalska-Metcalf IU, Balane-Bolivar C, Janicki SM, Greenberg RA: ATM-dependent chromatin changes silence transcription in cis to DNA double-strand breaks. Cell 2010, 141:970-981.

26. Gilson E, Geli V: How telomeres are replicated. Nat Rev Mol Cell Biol 2007, 8:825-838.

27. Bryan TM, Englezou A, Dalla-Pozza L, Dunham MA, Reddel RR: Evidence for an alternative mechanism for maintaining telomere length in human tumors and tumor-derived cell lines. Nat Med 1997, 3:1271-1274.

28. Henson JD, Reddel RR: Assaying and investigating Alternative Lengthening of Telomeres activity in human cells and cancers. FEBS Lett 2010, 584:3800-3811.

29. Heaphy CM, de Wilde RF, Jiao Y, Klein AP, Edil BH, Shi C, Bettegowda C, Rodriguez FJ, Eberhart CG, Hebbar S, et al.: Altered telomeres in tumors with ATRX and DAXX mutations. Science 2011, 333:425.

30. Jiao Y, Shi C, Edil BH, de Wilde RF, Klimstra DS, Maitra A, Schulick RD, Tang LH, Wolfgang CL, Choti MA, et al.: DAXXIATRX, MEN1, and mTOR pathway genes are frequently altered in pancreatic neuroendocrine tumors. Science 2011, 331:1199-1203.

*31. Lovejoy CA, Li W, Reisenweber S, Thongthip S, Bruno J, de Lange T, De S, Petrini JH, Sung PA, Jasin M, et al.: Loss of ATRX, Genome Instability, and an Altered DNA Damage Response Are Hallmarks of the Alternative Lengthening of Telomeres Pathway. PLoS Genet 2012, 8:e1002772. 
Comprehensive characterisation of 22 ALT cell lines, of which 19 had an alteration in the expression of ATRX and/or DAXX.

32. Schwartzentruber J, Korshunov A, Liu XY, Jones DT, Pfaff E, Jacob K, Sturm D, Fontebasso AM, Quang DA, Tonjes M, et al.: Driver mutations in histone H3.3 and chromatin remodelling genes in paediatric glioblastoma. Nature 2012, 482:226-231.

33. de Wilde RF, Heaphy CM, Maitra A, Meeker AK, Edil BH, Wolfgang CL, Ellison TA, Schulick RD, Molenaar IQ, Valk GD, et al.: Loss of ATRX or DAXX expression and concomitant acquisition of the alternative lengthening of telomeres phenotype are late events in a small subset of MEN-1 syndrome pancreatic neuroendocrine tumors. Mod Pathol 2012, 25:10331039.

34. Sakai A, Schwartz BE, Goldstein S, Ahmad K: Transcriptional and developmental functions of the H3.3 histone variant in Drosophila. Curr Biol 2009, 19:1816-1820.

35. Baumann C, Viveiros MM, De La Fuente R: Loss of maternal ATRX results in centromere instability and aneuploidy in the mammalian oocyte and preimplantation embryo. PLoS Genet 2010, 6.

Figure legends

Figure 1. (a) Microarray analysis of ATRX ChIP DNA enrichment from human erythroblasts across the $220 \mathrm{~kb}$ terminal region of chromosome $16 \mathrm{p}$ containing the $\alpha$ globin genes (HBM, HBA2, HBA1, HBQ). (b) A schematic showing the relationship of the $\psi \zeta$ VNTR (peak of ATRX binding) to the adjacent $\alpha$-globin genes. The relative 
expression of the genes within the cluster are shown comparing normal controls and individuals with ATR-X syndrome. (c) $\psi \zeta$ VNTR length was measured in ATR-X patients with $\alpha$ thalassemia and the average length of the two alleles was plotted against the degree of $\alpha$ thalassemia as measured by \% red cells showing $\mathrm{HbH}$ inclusions (adapted from [15]).

Figure 2. Hypothetical model of how ATRX may function to facilitate the replication of structured (G4) DNA and how in its absence the repair of double strand breaks by homologous recombination triggers the ALT pathway. 
a.

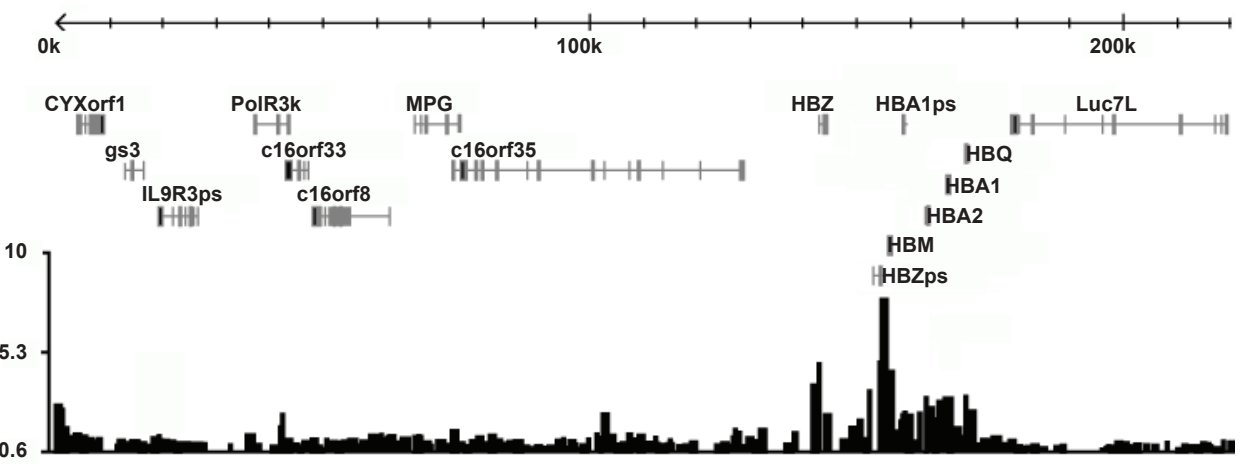

b.

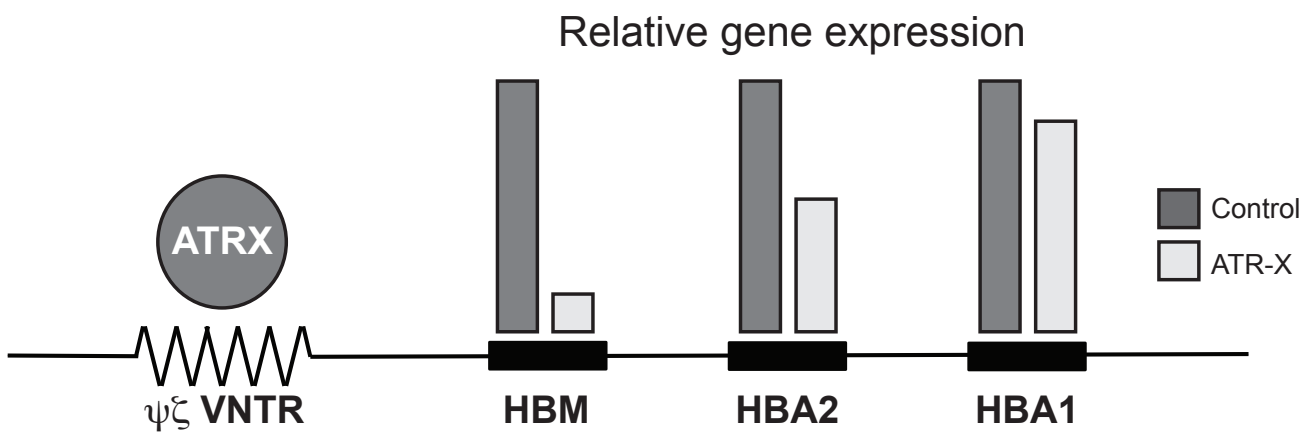

c.

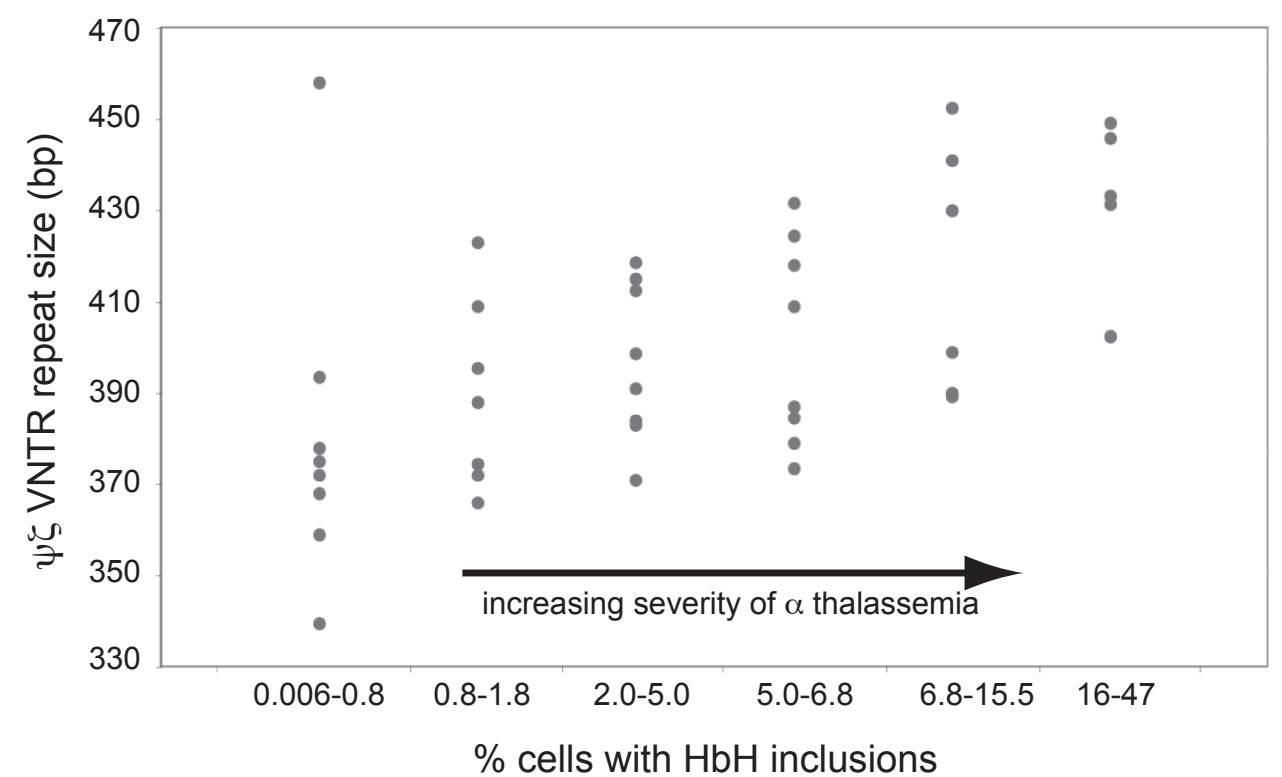




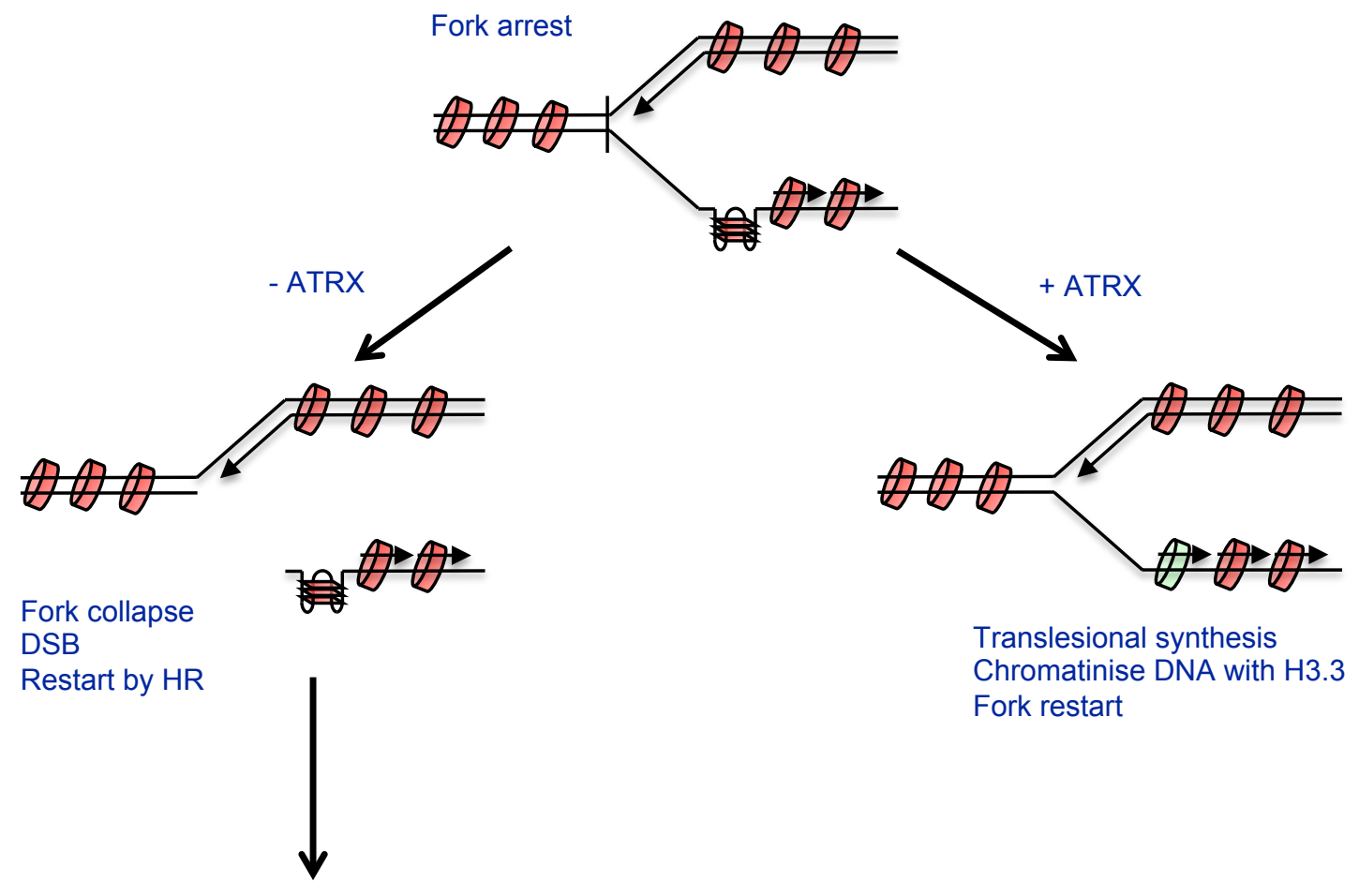

ALT pathway

in malignant cells 